\title{
Exosome Biomarkers Revolutionize \\ Preclinical Diagnosis of Neurodegenerative \\ Diseases and Assessment of Treatment \\ Responses in Clinical Trials
}

\author{
Dimitrios Kapogiannis
}

\begin{abstract}
Alzheimer's disease and other neurodegenerative diseases have long preclinical phases with active and progressively irreversible pathology. Therefore, biomarkers are essential for identifying patients early in the course of these diseases, when they may benefit the most from disease-modifying interventions. A limitation of biomarkers measured in the soluble phase of blood is their tenuous link to brain pathology. A new approach to biomarker discovery that addresses this limitation is deriving extracellular vesicles (EVs) enriched for neuronal and astrocytic origin from peripheral blood. EVs are membranous particles (subdivided into smaller exosomes and larger microvesicles) that are shed by all cells and found in all biofluids. Neuronal and astrocytic EVs have been implicated in the pathogenesis of several neurodegenerative diseases. Given their origin, neuronal and astrocytic enriched EVs harvested from blood can be used to interrogate brain pathologic processes previously inaccessible in vivo. In a long series of case control studies based on these EV subpopulations, we have identified candidate protein biomarkers for Alzheimer's disease and other neurodegenerative diseases. In GeNeDis 2018, an update of these studies and results from a validation study of these biomarkers in preclinical Alzheimer's disease will be presented. In addition, we will present results from studies demonstrating EV biomarker responses to experimental interventions. EV-based biomarkers are a valuable new tool that will enable researchers to test hypotheses in proof of concept studies with carefully selected participants at the preclinical phase, spearheading therapeutic discovery in neurodegenerative disease.
\end{abstract}

This research was supported entirely by the Intramural Research Program of the National Institute on Aging, NIH

D. Kapogiannis $(\bowtie)$

National Institute on Aging, NIH, Bethesda, MD, USA

Department of Neurology, Johns Hopkins University, Bethesda, MD, USA

e-mail: kapogiannisd@mail.nih.gov 